\title{
THE RESOLVENT AND RIESZ TRANSFORM ON CONNECTED SUMS OF MANIFOLDS WITH DIFFERENT ASYMPTOTIC DIMENSIONS
}

\author{
DANIEL NIX
}

\author{
(Received 8 April 2021; first published online 24 June 2021)
}

2020 Mathematics subject classification: primary 58B20; secondary 47A10, 47B06.

Keywords and phrases: analysis on manifolds, low energy resolvent, Riesz transform.

We consider the class of manifolds obtained by taking the connected sum of a finite number of $N$-dimensional Riemannian manifolds of the form $\left(\mathbb{R}^{n_{i}}, \delta\right) \times\left(\mathcal{M}_{i}, g\right)$, where $\mathcal{M}_{i}$ is a closed manifold, equipped with the product metric. The case of greatest interest is when the Euclidean dimensions $n_{i}$ are not all equal. This means that the ends have different 'asymptotic dimension', and it implies that these connected sums are nondoubling spaces.

In this thesis, we take a connected sum of two such product manifolds and assume that one of the Euclidean dimensions, $n_{i}$, is equal to 2 , which is a special case. Our approach is to construct the low energy resolvent and determine the asymptotics of the resolvent kernel as the energy tends to zero. The interesting feature of this case is the logarithmic behaviour of the resolvent kernel on the end with Euclidean dimension two. We express the Riesz transform in terms of the resolvent to show that it is bounded on $L^{p}$ for $1<p \leq 2$. This extends results from a paper of Hassell and Sikora [2] which considered connected sums of products of Euclidean spaces with closed manifolds, where each Euclidean space has a dimension of at least three.

We also analyse one-dimensional models of connected sums, which extends the work of Hassell and Sikora [1]. We consider the real line, which is equipped with a weighted Lebesgue measure with different power behaviour at $\pm \infty$. This space mimics higher-dimensional connected sums considered above in the sense of the Laplacian being restricted to radial functions. In the case where the negative half-line is weighted like $|r| d r$ and the positive half-line is weighted like $r^{d_{+}-1} d r, d_{+} \geq 3$, we show that the Riesz transform is $L^{p}$-bounded for the range $1<p \leq 2$, which agrees with our multidimensional case.

Thesis submitted to the Australian National University in December 2019; degree approved on 29 September 2020; supervisors Andrew Hassell and Adam Sikora.

(C) 2021 Australian Mathematical Publishing Association Inc. 


\section{References}

[1] A. Hassell and A. Sikora, 'Riesz transforms in one dimension', Indiana Univ. Math. J. 58(2) (2009), 823-852.

[2] A. Hassell and A. Sikora, 'Riesz transforms on a class of non-doubling manifolds', Comm. Partial Differential Equations 44(11) (2019), 1072-1099.

DANIEL NIX, Mathematical Sciences Institute,

The Australian National University, Canberra,

ACT 2601, Australia

e-mail: daniel.nix@anu.edu.au 